\title{
CYCLIC EXTENSIONS OF FREE PRO-P GROUPS AND $P$-ADIC MODULES
}

\author{
Anderson L. P. Porto and Pavel A. Zalesskit
}

\begin{abstract}
We prove a pro- $p$ version of the classical decomposition of a $\mathbb{Z}_{p}$-torsion free $\mathbb{Z}_{p} C_{p}$-module into indecomposable modules. We also describe some pro- $p \mathbb{Z}_{p} C_{p^{n-}}$ modules obtained from a semidirect product of a free pro- $p$ group $F$ and a cyclic group $C_{p^{n}}$ of automorphisms by factoring out the (closed) commutator subgroup $[F, F]$.
\end{abstract}

\section{Introduction}

Let $p$ be a prime number, $C_{p}$ a group of order $p, \mathbb{Z}_{p}$ the ring of $p$-adic integers and $\mathbb{Z}_{p} C_{p}$ the group ring. Let $M$ be a $\mathbb{Z}_{p}$-torsion free $\mathbb{Z}_{p} C_{p}$-module. If $M$ is finitely generated, then a classical result that plays a fundamental role in the theory of integral representations (cf. [2] or [4]) describes $M$ as a finite direct sum of cyclic modules of the form $\mathbb{Z}_{p} C_{p}, \mathbb{Z}_{p}$ and $J\left(\mathbb{Z}_{p} C_{p}\right)$, where $J\left(\mathbb{Z}_{p} C_{p}\right)$ is the augmentation ideal of $\mathbb{Z}_{p} C_{p}$.

Note that $\mathbb{Z}_{p} C_{p}$ is a local pro- $p$ ring, so a $\mathbb{Z}_{p} C_{p}$-module $M$ is finitely generated as a pro- $p \mathbb{Z}_{p} C_{p}$-module if and only if it is finitely generated as an abstract $\mathbb{Z}_{p} C_{p}$-module (see pp. 126-127 in Wilson [11]). If $M$ is infinitely generated then this is no longer the case, since an abstract infinitely generated $\mathbb{Z}_{p} C_{p}$-module is not necessarily compact so need not be pro- $p$. For infinitely generated abstract $\mathbb{Z}_{p} C_{p}$-modules the above result is not valid; $\mathbb{Q}_{p}$ considered as a trivial $\mathbb{Z}_{p} C_{p}$-module is not decomposable (since $\mathbb{Q}_{p}$ is not decomposable as a $\mathbb{Z}_{p}$-module).

We prove in this paper that surprisingly the classical result mentioned above holds for infinitely generated $\mathbb{Z}_{p}$-torsion free pro- $p \mathbb{Z}_{p} C_{p}$-modules.

Theorem A. Let $C=\langle x\rangle$ be a group of order $p$ and let $M$ be a $\mathbb{Z}_{p}$-torsion free pro- $p$ $\mathbb{Z}_{p} C$-module. Then $M$ decomposes as

$$
M=M_{T} \oplus M_{\theta_{p}} \oplus L,
$$

where $L$ is a free pro- $p \mathbb{Z}_{p} C$-submodule of $M, M_{T}$ is a trivial $\mathbb{Z}_{p} C$-module, $M_{\theta_{p}}$ is a free pro-p $\mathbb{Z}_{p}\left[\theta_{p}\right]$-module, where $\mathbb{Z}_{p}\left[\theta_{p}\right]$ is the quotient ring of $\mathbb{Z}_{p} C$ modulo the ideal $\left(\phi_{p}(x)\right)$ generated by the cyclotomic polynomial $\phi_{p}(x)=1+x+\ldots+x^{p-1}$ and $\theta_{p}$ is a root of $\phi_{p}(x)$. Moreover, $M_{T} \cong \bigoplus_{\gamma} \mathbb{Z}_{p}, M_{\theta_{p}} \cong \bigoplus_{\beta} J\left(\mathbb{Z}_{p} C\right)$ and $L \cong \bigoplus_{\phi} \mathbb{Z}_{p} C$ are profinite direct sums of pro- $p \mathbb{Z}_{p} C$-modules over Boolean spaces of indices $\gamma, \beta, \phi$, respectively.

Note that Theorem A can not be proved simply by using the projective limit argument since it is not clear why $M$ can be decomposed as an inverse limit of $\mathbb{Z}_{p}$-torsion free pro- $p \mathbb{Z}_{p} C_{p}$-modules. The existence of such a decomposition is a consequence of Theorem A.

Received by the editors May 21, 2012.

Key words and phrases. Virtually free pro- $p$ groups. Pro- $p$ modules. 
Now let $G=F \rtimes C_{p^{n}}$ be a pro- $p$ semidirect product of a free pro- $p$ group $F$ and a cyclic group $C_{p^{n}}$ of order $p^{n}$. The continuous action of $C_{p^{n}}$ on $F$ induces the structure

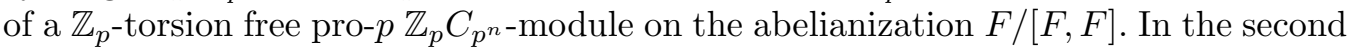
part of the paper, we study the structure of such pro- $p \mathbb{Z}_{p} C_{p^{n}}$-modules.

Theorem B. Let $G=U \rtimes H$ be a pro-p semidirect product of a free pro-p group $U$ and a cyclic group $H$ of order $p^{n}$. Suppose that the centralizers of all non-identity elements of finite order in $G$ are finite. Then

$$
U^{a b}:=U /[U, U] \cong\left(\bigoplus_{i \in(I, *)} J_{K_{i}}(H)\right) \bigoplus L
$$

is a profinite direct sum of pro-p $\mathbb{Z}_{p} H$-modules, where $(I, *)$ is a Boolean pointed space of indices, each $K_{i}$ is a subgroup of $H, J_{K_{i}}(H)$ is the kernel of the canonical epimorphism $\mathbb{Z}_{p} H \rightarrow \mathbb{Z}_{p}\left(H / K_{i}\right)$ and $L$ is a free pro-p $\mathbb{Z}_{p} H$-module.

In the proof, we use essentially Theorem 2.2 in Herfort-Zalesskii [5], which describes certain free-by-cyclic pro- $p$ groups as a free pro- $p$ product of normalizers of subgroups of order $p$ and some additional free factor.

If $n=1$ then we use Theorem A to prove that the abelianization $F^{a b}=F /[F, F]$ gives all possible $\mathbb{Z}_{p}$-torsion free pro- $p \mathbb{Z}_{p} C_{p}$-modules.

Theorem C. Let $M$ be a $\mathbb{Z}_{p}$-free pro-p $\mathbb{Z}_{p} C_{p}$-module. Then there exists a pro-p semidirect product $F \rtimes C_{p}$ of a free pro-p group $F$ and a group $C_{p}$ of order $p$ such that $F^{a b}$ is isomorphic to $M$ as a pro- $p \mathbb{Z}_{p} C_{p}$-module.

Note that for $n>1, F^{a b}$ does not give all possible $\mathbb{Z}_{p^{-}}$torsion free pro- $p \mathbb{Z}_{p} C_{p^{-}}$ modules, see Remark 4.5 in [8].

Basic results about profinite groups, rings and modules used in the paper can be found in [9] or [11], and for an account of injective and divisible modules see [10]. All groups and modules in the paper are pro- $p$, so all subgroups and submodules are closed and all homomorphisms are continuous; generation always means topological generation. Throughout the paper $p$ denotes a prime number, $\mathbb{Z}_{p}$ the ring of $p$-adic integers and $C_{p^{n}}$ denotes a cyclic group of order $p^{n}$. For a finite group $H$ we denote by $\mathbb{Z}_{p} H$ the group ring of $H$ over $\mathbb{Z}_{p}$ and $J(H)$ denotes the augmentation ideal of $\mathbb{Z}_{p} H$. If $K$ is a subgroup of $H$ we denote by $J_{K}(H)$ the kernel of the natural homomorphism $\mathbb{Z}_{p} H \longrightarrow \mathbb{Z}_{p}(H / K)$, where $\mathbb{Z}_{p}(H / K)$ is the free $\mathbb{Z}_{p}$-module over the coset space $H / K$. We use $\mathbb{Z}_{p}\left[\theta_{p}\right]$ to denote the quotient ring of $\mathbb{Z}_{p} C_{p}$ modulo the ideal $\left(\phi_{p}(x)\right)$ generated by the cyclotomic polynomial $\phi_{p}(x)=1+x+\cdots+x^{p-1}$ - that is, the ring obtained from $\mathbb{Z}_{p}$ by adding a primitive $p$ th root of unity. If $R$ is a ring with unity, denote by $R^{\times}$its group of units. We shall denote by $[A, B]$ the topological closure of the mutual commutator subgroup of subgroups $A$ and $B$ of a given group and by $F^{a b}=F /[F, F]$ the abelianization of a group $F$.

\section{Preliminary results}

Let $M$ be a pro- $p \mathbb{Z}_{p} H$-module. We say that a collection $\left\{M_{t}, t \in T\right\}$ of closed $\mathbb{Z}_{p} H$-submodules of $M$ indexed by a Boolean space $T$ is a continuous system of $\mathbb{Z}_{p} H$ submodules of $M$ if for each open neighbourhood $U$ of 0 in $M$, the set $T(U)=\{t \in$ $\left.T \mid M_{t} \subset U\right\}$ is open in $T$. 
Definition 2.1 ([6]). Let $M$ be a profinite $\mathbb{Z}_{p} H$-module, $T$ a Boolean space and $\left\{M_{t} \mid t \in T\right\}$ a continuous system of $\mathbb{Z}_{p} H$-submodules of $M$. We say that $M$ is a profinite direct sum of $M_{t}, t \in T$, notation $M=\bigoplus_{t \in T} M_{t}$ if:

(1) $M_{s} \cap M_{r}=\{0\} \forall r \neq s \in T$ and

(2) The following universal property holds: any given continuous map $\lambda: \bigcup_{t \in T}$ $M_{t} \longrightarrow K$ to a profinite $\mathbb{Z}_{p} H$-module $K$ such that each restriction $\lambda_{\mid M_{t}}$ : $M_{t} \longrightarrow K$ is a continuous $\mathbb{Z}_{p} H$-homomorphism, extends to a unique continuous $\mathbb{Z}_{p} H$-homomorphism $\bar{\lambda}: \bigoplus_{t \in T} M_{t} \longrightarrow K$.

The concept of a free pro- $p$ product of a continuous system of closed subgroups over a Boolean space is defined in a manner analogous to Definition 2.1.

Let $G$ be a pro- $p$ group having an open free pro- $p$ subgroup $F$. Then the set $\mathcal{T}$ of all subgroups of order $p$ in $G$ is a profinite space of indices, since it is the projective limit of corresponding finite discrete spaces of quotients $G / U$, where $U$ runs through the open normal subgroups of $G$ which are contained in $F$. Moreover, $G$ acts continuously on $\mathcal{T}$ by conjugation.

Theorem 2.2 (Theorem $2.2[5]$ ). Let $G \cong F \rtimes C_{p^{n}}$ be a cyclic extension of a free pro-p group $F$. Suppose $\mathcal{T} \longrightarrow \mathcal{T} / G$ admits a continuous section $\sigma$. Then

$$
G \cong\left(\coprod_{T \in \sigma(\mathcal{T} / G)} C_{G}(T)\right) \coprod \widetilde{F}
$$

is a free pro-p product of the centralizers $C_{G}(T)$ of groups $T$ of order $p$ over a Boolean space $\sigma(\mathcal{T} / G)$ of indices and a free pro-p subgroup $\widetilde{F}$ of $F$. Moreover, each $C_{G}(T)$ is a semidirect product of open free pro-p subgroup of $F$ by a finite cyclic group of order $p^{k}$, where $1 \leq k \leq n$.

Corollary 2.3. Suppose $C_{F}(t)=\{1\}$ for every torsion element $t \neq 1$ of $G$. Then $G=\left(\coprod_{i \in I} T_{i}\right) \amalg F(X)$ is a free pro-p product of groups $T_{i} \cong C_{p^{k_{i}}}$, where $1 \leq k_{i} \leq n$, $F(X)$ is a free pro-p group and $I$ is a profinite space.

Proof. Since in our case $F$ acts freely on the profinite space $\mathcal{T}$ of subgroups of order $p, \mathcal{T} \longrightarrow \mathcal{T} / F$ admits a continuous section $\sigma: \mathcal{T} / F \longrightarrow \mathcal{T}$ (see Lemma 5.6.5 in [9]). Put $I=\operatorname{Im}(\sigma)$. Since by hypothesis $C_{G}(T)$ is finite cyclic for each $T$, by Theorem 2.2 we get the required decomposition.

Remark 2.4. Since a torsion free abelian pro- $p$ group is free abelian (see Chapter 4 in $[9]$ ), $\mathbb{Z}_{p}$-torsion freeness is equivalent to $\mathbb{Z}_{p}$-freeness, thus we shall use this shorter term in the rest of the paper.

\section{The Heller-Reiner decomposition}

Lemma 3.1. The equation $\left(\theta_{p}-1\right) x=p z$ has a solution in any quotient ring $R$ of $\mathbb{Z}_{p}\left[\theta_{p}\right]$ for any $z \in R$. 
Proof. Since the maximal ideal of $\mathbb{Z}_{p}\left[\theta_{p}\right]$ is principal with $\theta_{p}-1$ being a generator (see Proposition $7.13[1])$, the solution exists in $\mathbb{Z}_{p}\left[\theta_{p}\right]$. Let $\phi: \mathbb{Z}_{p}\left[\theta_{p}\right] \rightarrow R$ be the canonical epimorphism. Denote by $\tilde{z}$ an element of $\mathbb{Z}_{p}\left[\theta_{p}\right]$ such that $\phi(\tilde{z})=z$. Then by the above $\left(\theta_{p}-1\right) x=p \tilde{z}$ has a solution $r$ in $\mathbb{Z}_{p}\left[\theta_{p}\right]$. Then $\phi(r)$ is a required solution.

Lemma 3.2. Let $M$ be a $\mathbb{Z}_{p}\left[\theta_{p}\right]$-module. Suppose $M$ is divisible as an abelian group. Then $M$ is a divisible $\mathbb{Z}_{p}\left[\theta_{p}\right]$-module.

Proof. It suffices to show that the multiplication by $\theta_{p}-1$ is an automorphism of $M$. In other words we need to show that for any $y \in M$ the equation $\left(\theta_{p}-1\right) x=y$ has a solution in $M$, since each element $a \in \mathbb{Z}_{p}\left[\theta_{p}\right]$ is of the form $\left(\theta_{p}-1\right)^{n} \cdot \epsilon$, with $\epsilon \in\left(\mathbb{Z}_{p}\left[\theta_{p}\right]\right)^{\times}$for some non-negative integer $n$ (see pp. 121 [1]). As $M$ is $p$-divisible, $y=p z$ for some $z \in M$. Let $\langle z\rangle$ be the submodule of $M$ generated by $z$. Then $\langle z\rangle$ as a cyclic module is isomorphic to some quotient ring of $\mathbb{Z}_{p}\left[\theta_{p}\right]$ (see Theorem 2.2 in [10]), so that the result follows from Lemma 3.1.

Lemma 3.3. Let $C=\langle x\rangle$ be a cyclic group of order $p$ and let $B$ be a $\mathbb{Z}_{p}$-free pro- $p$ $\mathbb{Z}_{p} C$-module. Suppose that $B$ is annihilated by $\phi_{p}(x)=1+x+\cdots+x^{p-1}$. Then $B$ is a free pro-p $\mathbb{Z}_{p}\left[\theta_{p}\right]$-module.

Proof. Consider the dual module $B^{*}=\operatorname{Hom}_{\mathbb{Z}_{p}}\left(B, \mathbb{Q}_{p} / \mathbb{Z}_{p}\right)$. Since $B$ is $\mathbb{Z}_{p}$-free, by Theorem 4.3.3 in $[9] B \cong \prod \mathbb{Z}_{p}$ and so $B^{*} \cong \bigoplus_{J} \operatorname{Hom}_{\mathbb{Z}_{p}}\left(\mathbb{Z}_{p}, \mathbb{Q}_{p} / \mathbb{Z}_{p}\right) \cong \bigoplus_{J} \mathbb{Q}_{p} / \mathbb{Z}_{p}$ as a $\mathbb{Z}_{p}$-module, where $J$ is some indexing set. Since $\mathbb{Q}_{p} / \mathbb{Z}_{p}$ is divisible it follows from Exercise 3.17 in [10] that $B^{*}$ is a divisible $\mathbb{Z}_{p}$-module. By Lemma $3.2 B^{*}$ is divisible as a $\mathbb{Z}_{p}\left[\theta_{p}\right]$-module and since $\mathbb{Z}_{p}\left[\theta_{p}\right]$ is a principal ideal domain (page 121 in [7]), it follows that $B^{*}$ is injective as a $\mathbb{Z}_{p}\left[\theta_{p}\right]$-module (cf. Theorem 3.24 in [10]). Therefore $B$ is a projective $\mathbb{Z}_{p}\left[\theta_{p}\right]$-module and as $\mathbb{Z}_{p}\left[\theta_{p}\right]$ is a local pro- $p$ ring, $B$ is a free pro- $p$ $\mathbb{Z}_{p}\left[\theta_{p}\right]$-module (see [11], pp. 127).

Lemma 3.4. Let $C=\langle x\rangle$ be a cyclic group of order $p$ and let $M$ be a $\mathbb{Z}_{p}$-free pro- $p$ $\mathbb{Z}_{p} C$-module. Let $\phi_{p}: M \longrightarrow M$ be the $\mathbb{Z}_{p} C$-homomorphism $m \longmapsto \phi_{p}(x) m$ and let $\pi$ be the canonical $\mathbb{Z}_{p} C$-epimorphism of $M$ onto $M / p M$. Then the kernel of $\pi \circ \phi_{p}$ is equal to $\left(M^{C} \oplus M_{p-1}\right)+p M$, where $M_{p-1}$ is the $\mathbb{Z}_{p} C$-submodule annihilated by the cyclotomic polynomial $\phi_{p}(x)$ and $M^{C}$ is the $\mathbb{Z}_{p} C$-submodule of fixed points for the action of $C$ on $M$.

Proof. Clearly $M^{C} \subset \operatorname{Ker}\left(\pi \circ \phi_{p}\right)$, because for all $m \in M^{C}$, we have $\phi_{p}(x) m=p m$ whence $\left(\pi \circ \phi_{p}\right)(m)=0$. On the other hand $\phi_{p}(x) M_{p-1}=\{0\}$, so $M_{p-1}$ is contained in the kernel of $\pi \circ \phi_{p}$. Thus, we must prove the converse containment. Suppose on the contrary that $\operatorname{Ker}\left(\pi \circ \phi_{p}\right) \not \subset\left(M_{p-1}+M^{C}+p M\right)$. In this case, there is an element $m \in \operatorname{Ker}\left(\pi \circ \phi_{p}\right) \backslash\left(M^{C}+M_{p-1}+p M\right)$. Since cyclic modules are only of the form $\mathbb{Z}_{p}$, $\mathbb{Z}_{p}\left[\theta_{p}\right]$ and $\mathbb{Z}_{p} C$ (see Theorem 2.6 in [4]), one has $\langle m\rangle \cong \mathbb{Z}_{p} C_{p}$, and so $\phi_{p}(x) m=p y$ for some $0 \neq y \in M$. It follows that $(x-1) \phi_{p}(x) m=(x-1) p y=0$, i.e. $p y \in M^{C}$. Since $M$ is $\mathbb{Z}_{p}$-free, it follows that $y \in M^{C}$. Then $\phi_{p}(x)(m-y)=p y-p y=0$ and so $m=(m-y)+y \in M_{p-1}+M^{C}$ as needed. The proof is finished.

Lemma 3.5. Let $C=\langle x\rangle$ be a cyclic group of order $p$ and let $M$ be a $\mathbb{Z}_{p}$-free pro- $p$ $\mathbb{Z}_{p} C$-module. Let $L$ be a free pro-p $\mathbb{Z}_{p} C$-submodule of $M$. Then $M^{C} /\left(M^{C} \cap L\right)$ is a free pro-p $\mathbb{Z}_{p}$-module and $M_{p-1} /\left(M_{p-1} \cap L\right)$ is a free pro-p $\mathbb{Z}_{p}\left[\theta_{p}\right]$-module. 
Proof. If $L=\{0\}$, there is nothing to prove. Suppose $L \neq\{0\}$. Since $L$ is $\mathbb{Z}_{p} C$-free, $L \cap M^{C}=L^{C}=\phi_{p}(x) L$ and $L_{p-1}:=L \cap M_{p-1}=(x-1) L$. Let $y \in M^{C} \backslash\left(M^{C} \cap L\right)$ be such that $p y \in M^{C} \cap L$. Then we can find an element $l$ of some free $\mathbb{Z}_{p} C$-basis for $L$ such that $p y$ belongs to the free cyclic pro- $p \mathbb{Z}_{p} C$-submodule $\langle l\rangle$. Namely, if $l_{0}$ is a generator of $L^{C}$ as a trivial $\mathbb{Z}_{p} C$-module and such that $p y \in\left\langle l_{0}\right\rangle$, then $l$ can be chosen arbitrarily such that $l_{0}=\phi_{p}(x) l$. Then the pro- $p \mathbb{Z}_{p} C$-submodule $\langle y, l\rangle$ of $M$ is finitely generated and so by the classical Heller-Reiner decomposition (cf. [4]) it decomposes as a direct sum of $\mathbb{Z}_{p} C$-submodules: $\langle y, l\rangle=\langle l\rangle \oplus L_{1}$, where $L_{1}$ is a trivial $\mathbb{Z}_{p} C$-submodule of $M^{C}$. Hence $\langle y, l\rangle /\left(M^{C} \cap\langle y, l\rangle\right)$ is $\mathbb{Z}_{p}$-torsion free, contradicting our assumptions on $y$. Thus $M^{C} /\left(M^{C} \cap L\right)$ is $\mathbb{Z}_{p}$-torsion free and so by Remark 2.4 is a free pro- $p \mathbb{Z}_{p}$-module. Similarly let $z \in M_{p-1} \backslash\left(L \cap M_{p-1}\right)$ be such that $p z \in L \cap M_{p-1}$. Then we can find an element $l^{\prime}$ of some free $\mathbb{Z}_{p} C$-basis for $L$ such that $p z$ belongs to the free cyclic pro- $p \mathbb{Z}_{p} C$-submodule $\left\langle l^{\prime}\right\rangle$. Namely, taking $l_{0}$ to be an element outside of the product $I M_{p-1}$ such that $p z \in\left\langle l_{0}\right\rangle$, where $I$ is the maximal ideal of $(x-1) \mathbb{Z}_{p} C$, one can choose $l^{\prime}$ to be any element element such that $(x-1) l^{\prime}=l_{0}$. Then $\left\langle z, l^{\prime}\right\rangle$ decomposes as a direct sum of $\mathbb{Z}_{p} C$-submodules: $\left\langle l^{\prime}\right\rangle \oplus R$, where $R$ is a free cyclic $\mathbb{Z}_{p}\left[\theta_{p}\right]$-module (see cf. [4]). Hence $\left\langle z, l^{\prime}\right\rangle /\left(M_{p-1} \cap\left\langle z, l^{\prime}\right\rangle\right)$ is $\mathbb{Z}_{p}$-torsion free, contradicting our assumptions on $z$. Thus, $M_{p-1} /\left(M_{p-1} \cap L\right)$ is $\mathbb{Z}_{p}$-torsion free and so by Lemma 3.3 is a free pro- $p \mathbb{Z}_{p}\left[\theta_{p}\right]$-module.

Theorem A. Let $C=\langle x\rangle$ be a group of order $p$ and let $M$ be a $\mathbb{Z}_{p}$-free pro-p $\mathbb{Z}_{p} C$ module. Then $M$ decomposes as

$$
M=M_{T} \oplus M_{\theta_{p}} \oplus L,
$$

where $L$ is a free pro-p $\mathbb{Z}_{p} C$-submodule of $M, M_{T}$ is a trivial $\mathbb{Z}_{p} C$-module and $M_{\theta_{p}}$ is a free pro- $p \mathbb{Z}_{p}\left[\theta_{p}\right]$-module. Moreover, $M_{T} \cong \bigoplus_{\gamma} \mathbb{Z}_{p}, M_{\theta_{p}} \cong \bigoplus_{\beta} J\left(\mathbb{Z}_{p} C\right)$ and $L \cong$ $\bigoplus_{\phi} \mathbb{Z}_{p} C$ as pro-p $\mathbb{Z}_{p} C$-modules, where $\gamma, \beta, \phi$ are Boolean space of indices.

Proof. Consider $M / p M$ as a pro- $p \mathbb{F}_{p} C$-module. Then $\widetilde{M}:=\operatorname{Hom}\left(M / p M, \mathbb{F}_{p}\right)$ is a discrete $\mathbb{F}_{p} C$-module. Consider the family of all injective $\mathbb{F}_{p} C$-submodules of $M / p M$ partially ordered by inclusion. Since $\mathbb{F}_{p} C$ is a Noetherian ring, it follows from Theorem 4.10 in [10], that the direct limit of such injective $\mathbb{F}_{p} C$-submodules is injective, so that by Zorn's Lemma there exists a maximal injective $\mathbb{F}_{p} C$-submodule $\widetilde{L}$ in $\widetilde{M}$. Now $\widetilde{L}$ has a complement $\widetilde{K}$ in $\widetilde{M}$, i.e. $\widetilde{M}=\widetilde{L} \oplus \widetilde{K}$. By Pontryagin duality (see [3], pp. 332)

$$
M / p M \cong \operatorname{Hom}\left(\widetilde{L} \oplus \widetilde{K}, \mathbb{F}_{p}\right) \cong \operatorname{Hom}\left(\widetilde{L}, \mathbb{F}_{p}\right) \oplus \operatorname{Hom}\left(\widetilde{K}, \mathbb{F}_{p}\right)
$$

Put $\bar{L}:=\operatorname{Hom}\left(\widetilde{L}, \mathbb{F}_{p}\right)$ and $\bar{K}:=\operatorname{Hom}\left(\widetilde{K}, \mathbb{F}_{p}\right)$. We shall identify $\bar{L}$ with the copy of $\bar{L}$ in $M / p M$, and do the same with $\bar{K}$. Then $\bar{L}$ is projective $\mathbb{F}_{p} C$-submodule of $M / p M$. As $\mathbb{F}_{p} C$ is a local pro- $p$ ring (see Proposition 7.5.3, pp.126 in [11]) $\bar{L}$ is a free $\mathbb{F}_{p} C$-submodule of $M / p M$ (see Corollary 7.5.4, pp. 127 in [11]).

Let $\pi: M \longrightarrow M / p M$ be the natural epimorphism. By Proposition 2.2.2 in RibesZalesskii [9], $\pi$ admits a continuous section $\delta: M / p M \longrightarrow M$ with $\delta(0+M)=0$. Consider a profinite space $\Omega$ of free generators of $\bar{L}$ converging to 0 . Put $\mathcal{X}=\delta(\Omega)$. Let $L$ be the closed $\mathbb{Z}_{p} C$-submodule of $M$ topologically generated by $\mathcal{X}$. Then $L$ is a free pro- $p \mathbb{Z}_{p} C$-submodule on $\mathcal{X}$. Indeed, let $A$ be a free pro- $p \mathbb{Z}_{p} C$-module on $\mathcal{X}$ and $f: A \longrightarrow L$ be the $\mathbb{Z}_{p} C$-epimorphism induced by sending $\mathcal{X}$ identically to its copy in $L$. Then as a pro- $p$ group $A$ is free pro- $p$ abelian on the basis $C \mathcal{X}$. Since $\bar{L}$ is a free 
$\mathbb{F}_{p} C$-module on $\Omega$, it is an elementary abelian pro- $p$ group on $C \Omega$. This shows that the kernel of $f$ is contained in the Frattini subgroup $\Phi(A)$. But a homomorphism of free abelian pro- $p$ groups with the kernel in the Frattini subgroup is an isomorphism. Thus $f$ is an isomorphism.

Let $M^{C}$ be the pro- $p \mathbb{Z}_{p} C$-submodule of fixed points in $M$, i.e., the closed $\mathbb{Z}_{p} C$ submodule of $M$, annihilated by $(x-1)$. Consider the natural epimorphism $s: M^{C} \longrightarrow$ $M^{C} /\left(L \cap M^{C}\right)$. As by Lemma $3.5 M^{C} /\left(L \cap M^{C}\right)$ is free pro- $p$ as a $\mathbb{Z}_{p}$-module, it follows that $M^{C}=\left(L \cap M^{C}\right) \oplus U$, where $U$ is a complement for $L \cap M^{C}$.

Now consider the pro- $p \mathbb{Z}_{p} C$-submodule $M_{p-1}$ of $M$ annihilated by $\left(\phi_{p}(x)\right)$. By Lemma 3.3, $M_{p-1}$ is a free pro- $p \mathbb{Z}_{p}\left[\theta_{p}\right]$-module. Consider the natural epimorphism $r: M_{p-1} \longrightarrow M_{p-1} /\left(L \cap M_{p-1}\right)$. As by Lemma $3.5 M_{p-1} /\left(L \cap M_{p-1}\right)$ is a free pro- $p \mathbb{Z}_{p}\left[\theta_{p}\right]$-module, we have $M_{p-1}=\left(L \cap M_{p-1}\right) \oplus V$, where $V$ is a pro- $p \mathbb{Z}_{p}\left[\theta_{p}\right]$ submodule of $M_{p-1}$.

Thus $L \cap V=\{0\}$ and so $L+V=L \oplus V$. As $M^{C} \cap M_{p-1}=\{0\}$ one has $U \cap(L+V)=\{0\}$ so that $L+U+V=L \oplus U \oplus V$. We want to show that $M=L \oplus U \oplus V$ as a pro- $p \mathbb{Z}_{p} C$-module.

It suffices to prove that $M=\left\langle M^{C}, M_{p-1}, L\right\rangle$ as a $\mathbb{Z}_{p} C$-module, since $U \oplus V \oplus L$ contains $L \cap M^{C}, U, V, L \cap M_{p-1}$ and $M_{p-1}=\left(L \cap M_{p-1}\right) \oplus V, M^{C}=\left(L \cap M^{C}\right) \oplus U$.

Consider the following diagram:

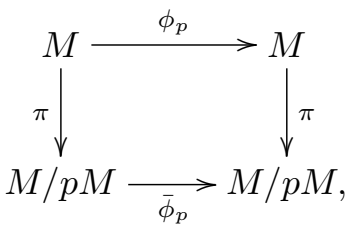

where $\phi_{p}: M \longrightarrow M$ is a $\mathbb{Z}_{p} C$-homomorphism sending $m \longmapsto \phi_{p}(x) m, \pi$ is the canonical $\mathbb{Z}_{p} C$-epimorphism of $M$ to $M / p M$ and $\overline{\phi_{p}}: M / p M \longrightarrow M / p M$ is a $\mathbb{Z}_{p} C$ homomorphism sending $\bar{m} \longmapsto \phi_{p}(x) \bar{m}$. Clearly the diagram is commutative.

Recall that $\bar{L}$ is a free $\mathbb{F}_{p} C$-submodule of $M / p M$ and $M / p M \cong \bar{L} \oplus \bar{K}$, where $\bar{K}$ is a $\mathbb{F}_{p} C$-complement of $\bar{L}$ in $M / p M$. By the commutativity of the above diagram the preimage of $\bar{K}$ in $M$ is contained in $\operatorname{Ker}\left(\pi \circ \phi_{p}\right)$. By Lemma 3.4, $M=\left\langle L, \operatorname{Ker}\left(\pi \circ \phi_{p}\right)\right\rangle=\left\langle L, M^{C}+M_{p-1}+p M\right\rangle$ as a free abelian pro- $p$ group. As $p M=\Phi(M)$ is the Frattini subgroup of the abelian pro- $p$ group $M$, we have $M=$ $L+M^{C}+M_{p-1}$. Finally put $M_{T}=U$ and $M_{\theta_{p}}=V$.

The second part of the statement follows from the definition of a free module on a Boolean space of indices (see page 108 in [6]).

\section{Finite centralizers of torsion elements}

Theorem B. Let $G=U \rtimes H$ be a pro-p semidirect product of a free pro-p group $U$ and a cyclic group $H$ of order $p^{n}$. Suppose that the centralizers of all non-identity elements of finite order in $G$ are finite. Then

$$
U^{a b}:=U /[U, U] \cong\left(\bigoplus_{i \in(I, *)} J_{K_{i}}(H)\right) \bigoplus L
$$


is a profinite direct sum of pro-p $\mathbb{Z}_{p} H$-modules, where $(I, *)$ is a Boolean pointed space of indices, the $K_{i}$ are subgroupss of $H, J_{K_{i}}(H)$ is the kernel of the canonical epimorphism $\mathbb{Z}_{p} H \rightarrow \mathbb{Z}_{p}\left(H / K_{i}\right)$ and $L$ is a free pro-p $\mathbb{Z}_{p} H$-module.

Proof. By Corollary 2.3, $G=\left(\coprod_{i \in I} T_{i}\right) \amalg F(X)$ is a free pro- $p$ product of groups $T_{i} \cong C_{p^{k_{i}}}$, where $1 \leq k_{i} \leq n, F(X)$ is a free pro- $p$ subgroup of $U$ and $I$ is a profinite index space. By Proposition 4.9 in [6], $H$ is conjugate to some finite free factor in $G$. Thus, we may assume that $H=T_{*} \cong C_{p^{n}}$ for some $* \in I$. Let $\phi: G \longrightarrow H$ be the endomorphism identical on $H$ and having $U$ as the kernel.

By Corollary 3.3.10 in Ribes-Zalesskii [9], we have $F(X)=\underset{\beta \in B}{\lim _{\beta}} F\left(X_{\beta}\right)$, where $X_{\beta}$ runs through the collection of all finite quotient sets of $X$ and $F\left(X_{\beta}\right)$ is a free pro- $p$ group with finite base $X_{\beta}$. Let $I=\varliminf_{\kappa \in K}^{\lim } I_{\kappa}$ be a decomposition of $I$ as an inverse limit of finite spaces $I_{\kappa}$ such that $\pi_{\kappa}(i)=\pi_{\kappa}(j)$ only if $\phi\left(T_{i}\right)=\phi\left(T_{j}\right)$, where $\pi_{\kappa}: I \longrightarrow I_{\kappa}$ is the $\kappa$ th projection. Choose a generator for every subgroup of $H$ and let $S$ be the set of these generators. Then for every $i \in I$, there is a unique generator $t_{i}$ of $T_{i}$ such that $\phi\left(t_{i}\right) \in S$ and the set of all these generators is homeomorphic to $I$. Note that a projection $\pi_{\kappa}$ induces the homomorphisms $\coprod_{i \in I} T_{i} \longrightarrow \coprod_{i_{\kappa} \in I_{\kappa}} T_{i_{\kappa}}$ that identifies $t_{i}$ with $t_{j}$ whenever $\pi_{\kappa}(i)=\pi_{\kappa}(j)$.

This gives the inverse limit decomposition

$$
\begin{aligned}
G & \cong\left[\lim _{\kappa \in K}\left(\coprod_{i_{\kappa} \in I_{\kappa}} T_{i_{\kappa}}\right)\right] \coprod\left(\lim _{\beta \in B} F\left(X_{\beta}\right)\right) \\
& \cong \lim _{\kappa \in K, \beta \in B}\left[\left(\coprod_{i_{\kappa} \in I_{\kappa}} T_{i_{\kappa}}\right) \coprod F\left(X_{\beta}\right)\right]
\end{aligned}
$$

(cf. Lemma 9.1.5 in Ribes-Zalesskii [9]).

Note that $\phi$ factors via the epimorphisms

$$
f_{\kappa, \beta}:\left(\coprod_{i_{\kappa} \in I_{\kappa}} T_{i_{\kappa}}\right) \coprod F\left(X_{\beta}\right) \rightarrow H
$$

and we denote by $U_{\kappa, \beta}$ its kernel.

Then by Corollary 1.1.8 in Ribes-Zalesskii [9], $U=\lim _{K \times B} U_{\kappa, \beta}$. It follows that $U^{a b} \cong \varliminf_{K \times B} U_{\kappa, \beta}^{a b}$, where $U_{\kappa, \beta}^{a b}:=U_{\kappa, \beta} /\left[U_{\kappa, \beta}, U_{\kappa, \beta}\right]$

Put $*_{\kappa}$ to be the image of $*$ in $I_{\kappa}$. By Proposition 3.3 in [8]

$$
U_{\kappa, \beta}^{a b} \cong\left(\bigoplus_{i_{\kappa} \in I_{\kappa} \backslash\left\{*_{k}\right\}} J_{K_{i_{\kappa}}}(H)\right) \bigoplus L_{\kappa, \beta}
$$

as a pro- $p \mathbb{Z}_{p} H$-module, where $L_{\kappa, \beta}$ is a free pro- $p \mathbb{Z}_{p} H$-module with finite base, $J_{K_{i_{\kappa}}}(H)$ is the kernel of $\mathbb{Z}_{p} H \rightarrow \mathbb{Z}_{p}\left(H / K_{i_{\kappa}}\right)$. Moreover, it follows from the proof there that $L_{\kappa, \beta}$ has the image of $X_{\beta}$ in $U_{\kappa, \beta}^{a b}$ as a free $\mathbb{Z}_{p} H$-basis and that $J_{K_{i_{\kappa}}}(H)$ is generated by the images of the elements $t_{*_{\kappa}} t_{i_{\kappa}}^{-1}$ in the abelianization of $U_{\kappa, \beta}$, where 
$t_{*_{\kappa}} \in T_{*_{\kappa}} \cong H, t_{i_{\kappa}} \in T_{i_{\kappa}}$ with $U_{\kappa, \beta} t_{i_{\kappa}}=U_{\kappa, \beta} t_{*_{\kappa}}$. Note that since $I_{\kappa}$ is finite discrete space, $I_{k} \backslash\left\{*_{k}\right\}=\left(I_{k}, *_{\kappa}\right)$ and so

$$
\bigoplus_{i_{\kappa} \in I_{\kappa} \backslash\left\{*_{k}\right\}} J_{K_{i_{\kappa}}}(H)=\bigoplus_{i_{\kappa} \in\left(I_{\kappa}, *_{\kappa}\right)} J_{K_{i_{\kappa}}}(H)
$$

This means that the decomposition

$$
U_{\kappa, \beta}^{a b} \cong\left(\bigoplus_{i_{\kappa} \in\left(I_{\kappa}, *_{\kappa}\right)} J_{K_{i_{\kappa}}}(H)\right) \bigoplus L_{\kappa, \beta}
$$

is coherent with the inverse system for $U^{a b}$ and so by the commutation property between projective limits and profinite direct sums (see Proposition 1.6 on pp. 100 combined with 3.1 on page 107 in [6] ), we have

$$
U^{a b} \cong \lim _{\kappa \in K}\left[\bigoplus_{i_{\kappa} \in\left(I_{\kappa}, *_{\kappa}\right)} J_{K_{i_{\kappa}}}(H)\right] \bigoplus \underset{\beta \in B}{\lim _{\beta}} L_{\kappa, \beta} \cong \bigoplus_{i \in(I, *)} J_{K_{i}}(H) \bigoplus L
$$

which is the desired profinite direct sum, where $L$ is a free pro- $p \mathbb{Z}_{p} H$-module and $(I, *)=\lim _{\kappa \in K}\left(I_{\kappa}, *_{\kappa}\right)$.

Remark 4.1. The proof shows that $L$ is a free pro- $p \mathbb{Z}_{p} H$-module with closed free base $X[U, U] /[U, U]$.

The following corollary is a generalization of Lemma 3.1 in [8].

Corollary 4.2. If $T_{i} \cong C_{p^{n}}$, for all $i \in I$, then

$$
U^{a b} \cong \bigoplus_{i \in(I, *)} J(H) \bigoplus L
$$

where $L$ is a free pro-p $\mathbb{Z}_{p} H$-module and $J(H)$ is the augmentation ideal of $\mathbb{Z}_{p} H$.

Corollary 4.3. With the hypotheses of Theorem $B, H$ acts faithfully on $U^{a b}:=$ $U /[U, U]$.

Proof. The proof is the same as in Corollary 3.4 in Porto-Zalesskii [8], pp. 229.

Corollary 4.4. The $\mathbb{Z}_{p} H$-module $U^{a b}$ of Theorem $B$ is indecomposable as a $\mathbb{Z}_{p} H$ module if and only if $G$ has not more than two free factors and $|X| \leq 1$.

Proof. By Proposition 2.1 in [8], $J_{K_{i}}(H)$ is indecomposable $\mathbb{Z}_{p} H$-module for every $i \in(I, *)$. Hence the result follows from Theorem B.

Now we are ready to prove

Theorem C. Let $M$ be a $\mathbb{Z}_{p}$-free pro-p $\mathbb{Z}_{p} C_{p}$-module. Then there exists a pro-p semidirect product $F \rtimes C_{p}$ of a free pro-p group $F$ and a group $C_{p}$ of order $p$ such that $F^{a b}$ is isomorphic to $M$ as a pro-p $\mathbb{Z}_{p} C_{p}$-module. 
Proof. Let $c$ be a generator of $C_{p}$. By Theorem A, $M$ decomposes as

$$
M=M_{T} \oplus M_{\theta_{p}} \oplus L,
$$

where $L$ is a free pro- $p \mathbb{Z}_{p} C_{p^{-}}$-submodule of $M, M_{T}$ is a trivial pro- $p \mathbb{Z}_{p} C_{p}$-module and $M_{\theta_{p}}$ is a free pro- $p \mathbb{Z}_{p}\left[\theta_{p}\right]$-module; let $X, Y, Z$ be free profinite bases of $M_{T}, M_{\theta_{p}}, L$, respectively. Put $Y_{0}:=\bigcup_{j=0}^{p-2} c^{j} Y, Z_{0}:=\bigcup_{t=0}^{p-1} c^{t} Z$ and $W:=X \cup Y_{0} \cup Z_{0}$. Let $F=F(W)$ be the free pro- $p$ group on $W$. Define a pro- $p$ semidirect product $F \rtimes C_{p}$ putting for all $x \in X: x^{c}=x$; for each $y \in Y:\left(c^{k} y\right)^{c}=c^{k+1} y$ for $0 \leqslant k \leqslant p-3$ and $\left(c^{p-2} y\right)^{c}=\left(\prod_{r=0}^{p-2} c^{r} y\right)^{-1}$; and for all $z \in Z:\left(c^{s} z\right)^{c}=c^{s+1} z$ where $0 \leqslant s \leqslant p-1$; to be the action on the elements of the basis $W$ and extending it to the action on $F$ by the universal property of $F$. Then $F(X)^{a b} \cong M_{T}, F\left(Y_{0}\right)^{a b} \cong M_{\theta_{p}}$ and $F\left(Z_{0}\right)^{a b} \cong L$ as pro- $p \mathbb{Z}_{p} C_{p}$-modules, so that

$$
F^{a b} \cong F(X)^{a b} \oplus F\left(Y_{0}\right)^{a b} \oplus F\left(Z_{0}\right)^{a b} \cong M .
$$

\section{Acknowledgments}

The first author was partially supported by FAPEMIG, and the second author by CAPES and CNPq.

\section{References}

[1] J.W.S. Cassels and A. Frohlich, Algebraic number theory, Academic Press, London, New York, 1967.

[2] C.W. Curtis and I. Reiner, Methods of representation theory - with applications to finite groups and ordes, Wiley, New York, 1981.

[3] J. Flood, Pontryagin duality for topological modules, Proc. Amer. Math. Soc. 75(2) (1979), 329-333.

[4] A. Heller and I. Reiner, Representations of cyclic groups in ring of integers I, Ann. Math. 76(2) (1962), 73-92.

[5] W.N. Herfort and P.A. Zalesskii, Cyclic extensions of free pro-p groups, J. Algebra 216 (1999), 511-547.

[6] O.V. Mel'nikov, Subgroups and homology of free products of profinite groups, Math. USSR, Iz. 34 (1990), 97-119.

[7] J. Neukirch, Algebraic Number Theory, Springer, Berlin-Heidelberg, New York, 1999.

[8] A.L.P. Porto and P.A. Zalesskii, Free-by-finite pro-p groups and p-adic integral representations, Arch. Math. 97 (2011), 225-235.

[9] L. Ribes and P.A. Zalesskii, Profinite groups, 40, Springer-Verlag, Berlin-Heidelberg, New York, 2nd ed., 2010.

[10] J.J. Rotman, An introduction to homological algebra, Academic Press, University Illinois, Urbana, Illinois, 1979.

[11] J.S. Wilson, Profinite groups, Clarendon Press, Oxford, 1998.

Instituto de Ciência e Tecnologia (ICT), UFVJM, 39100-000, Diamantina-MG, Brazil

E-mail address: ander.porto@ict.ufvjm.edu.br

Departamento de Matemática, Universidade de Brasília, 70910-900, Brasília-DF, Brazil

E-mail address: pz@mat.unb.br 
